\title{
Flow Field around the Badminton Shuttlecock during Flipping Motion
}

\author{
Y. Sakurai ${ }^{1}$, K. Nakagawa ${ }^{2}$ and H. Hasegawa ${ }^{1}$ \\ ${ }^{1}$ Graduate School of Utsunomiya University, Utsunomiya, Japan \\ ${ }^{2}$ Utsunomiya University, Utsunomiya, Japan
}

\begin{abstract}
Badminton is one of the most popular sports in the world. The shuttlecock is used in badminton game has the unique shape. The shuttlecock is truncated cone-shaped and consists of a cork, gaps and a skirt portion. The shuttlecock has aerodynamic properties which differ from the ball used in other racquet sports. As an example of unique aerodynamic property, the shuttlecock shows high deceleration. It is known that the initial velocity immediately after smashing may reach up to $137 \mathrm{~m} / \mathrm{s}(493 \mathrm{~km} / \mathrm{h})$ at maximum. The velocities of the shuttlecock are reduced from the initial velocity of $67 \mathrm{~m} / \mathrm{s}$ to the terminal velocity of approximately $7 \mathrm{~m} / \mathrm{s}$ for approximately $0.6 \mathrm{~s}$ (Hubbard et al. 1997). In addition, turnover refers to the flipping experienced by a shuttlecock when undergoing heading change from nose pointing against the flight path at the moment of impact and a shuttlecock indicates the aerodynamically stable feature for the flip movement just after impact (Cohen et al. 2015). The turnover stability of a series of feather and synthetic shuttlecocks was measured to compare the performance of synthetic shuttlecocks to that of feather shuttlecocks (Calvin et al. 2013). The turnover stability of the shuttlecock is investigated through experiment and simulation, and the angular response of the shuttlecock in turnover was modelled and studied (Calvin et al. 2015). Furthermore, it was reported that the aerodynamic stability of the shuttlecock during flip movement was affected by gaps of the shuttlecock skirt in a previous study (Nakagawa et al. 2017). However, the mechanism of turnover stability of the shuttlecock has not been fully understood. The purpose of this study is to investigate the unsteady flow field around the shuttlecock during flip movements. In the present, we simulated the flipping motion by wind tunnel experiments and visualized the flow field around the shuttlecock by a PIV technique.

Figure 1 shows the schematic diagram of experimental apparatus and the experimental setup for the flow visualization system. The test section's dimensions were $0.7 \mathrm{~m} \times 0.7 \mathrm{~m}$ and the freestream turbulence intensity was less than $1 \%$ within the operation range. The origin of the coordinate system is defined as the center of mass of the shuttlecock. The velocity is denoted by the components $(u, v, w)$ in the directions $(X$, $Y, Z$ ). The test model is YONEX feather shuttlecock (NEW OFICIAL, No4, YONEX Co. Ltd.), which is the official choice for the world's leading international tournaments. The PIV system in this experiment mainly consists of a high-speed camera (MEMRECAM HX-6, nac Image Technology Inc.) and a YAGLaser (LDP-100MQG). The wind speed was set at $10 \mathrm{~m} / \mathrm{s}$ corresponding to the Reynolds number $R e$ based on the shuttlecock skirt diameter of $4.3 \times 10^{4}$. The angle of attack $\alpha=0^{\circ}$ indicates the state that the cork of the shuttlecock was set in the upstream direction in this experiment.
\end{abstract}

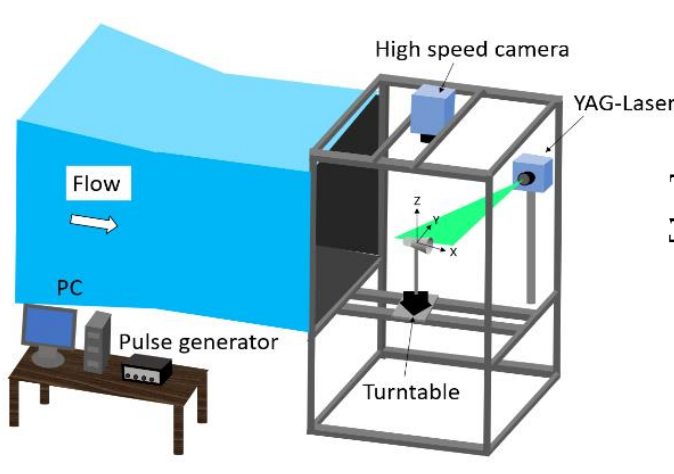

Figure 1: An overview of the experimental set up

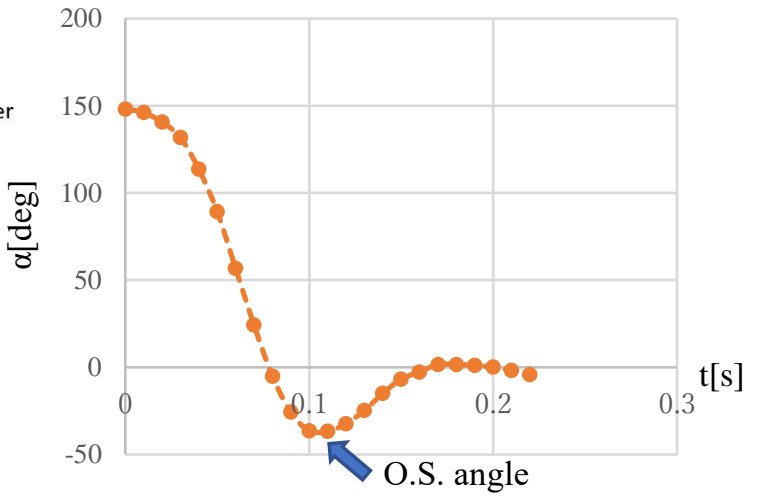

Figure 2: Angular response of shuttlecock in turnover process 
The angles of attack of the shuttlecock versus elapse time during flip movements is shown in Figure 2. The shuttlecock's angle of attack is estimated by the high-speed camera. The initial angle of attack in the flipping experiments was set as $\alpha=145^{\circ}$. The shuttlecock flips in the counter clockwise rotation beyond $\alpha=0^{\circ}$ and changes to rotational direction (clockwise) at overshoot angle $\alpha=-38.5^{\circ}$. To eliminate any discrepancies arising from externalities, each shuttlecock was tested 5 times, and the same tendency as the experimental result was confirmed.

Figure 3 shows the unsteady flow fields around the shuttlecock in the turnover process. The results are phase-averaged by synchronizing the angle of attack of the shuttlecock over four measurements. At $\alpha=0^{\circ}$, the flow through the shuttlecock gap is observed in the near-wake region of the shuttlecock skirt, and the flow vectors tilt to the right. At $\alpha=-24.4^{\circ}$, the flow through the gap is divided into two streams, one that merges into the velocity shear layer behind the right side of the shuttlecock skirt and the other that flows to the rearward of the shuttlecock, and this flow produces a counterclockwise flow field behind the shuttlecock skirt at $\alpha=-32.8^{\circ}$ (circle in Figure3(c)). The image of the flow transition after the overshoot is depicted in Figure 4. The flow through the gap which generates the vortex is related to the turnover stability.

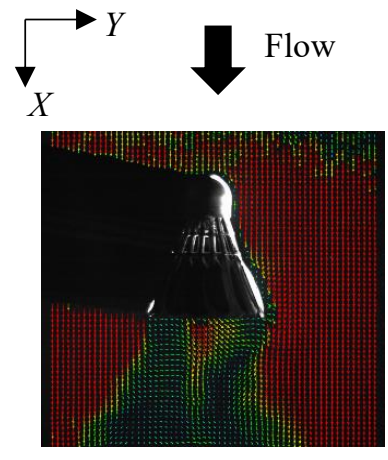

(a) $\alpha=0^{\circ}$ $t=0.069 \mathrm{~s}$

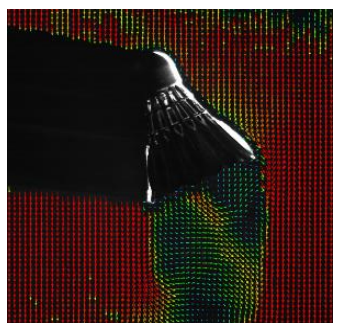

(b) $\alpha=-24.4^{\circ}$ $t=0.081 \mathrm{~s}$ velocity $[\mathrm{m} / \mathrm{s}]$

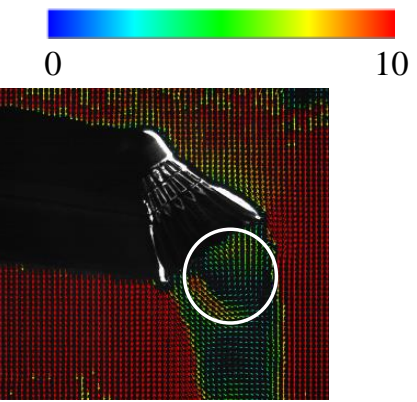

(c) $\alpha=-32.8^{\circ}$

$t=0.088 \mathrm{~s}$

Figure 3: Flow vectors around the shuttlecock in the turnover process

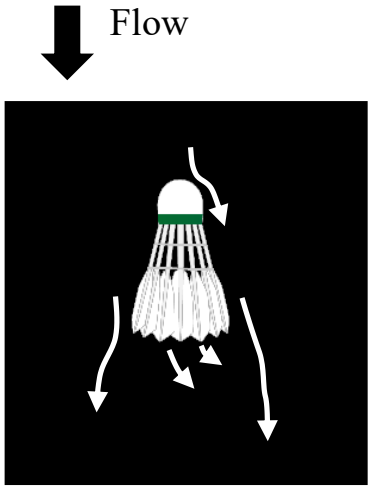

(a) $\alpha=0^{\circ}$

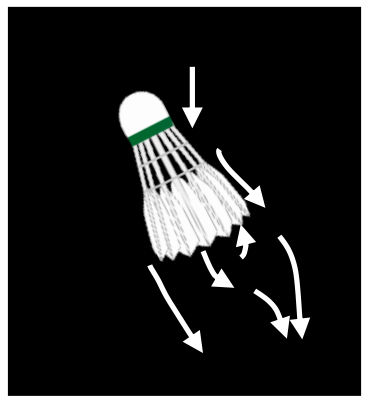

(b) $\alpha=-24.4^{\circ}$

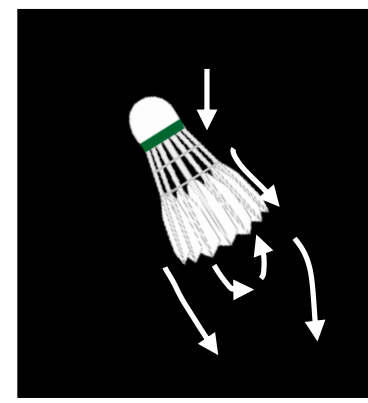

(c) $\alpha=-32.8^{\circ}$

Figure 4: The cartoon depicting of flow field around the shuttlecock during flip movement

\section{Reference}

Hubbard M. and Alison J. Cooke., Spin dynamics of the badminton shuttlecock. 6th International Symposium on Computer Simulation in Biomechanics. 1997, pp. 42-43.

Nakagawa K., Hasegawa H., Murakami M., and Obayashi S., Aerodynamic stability of a badminton shuttlecock. Transactions of the JSME (in Japanese), Vol.83, No856, 2017.

Calvin S.H. Lin., C. K. Chua and J. H. Yeo, Turnover stability of shuttlecocks - Transient angular response and impact deformation of feather and synthetic shuttlecocks. Procedia Engineering, 60, 2013, pp. 106111.

Cohen C., Texier B.D., Quere D. and Clanet C., The physics of badminton, 2015, New Journal of Physics, 17,063001

Calvin S.H. Lin., CK Chua. and JH Yeo., Badminton shuttlecock stability: Modelling and simulating the angular response of the turnover, 2015, Proc. IMechE Part P: J Sports Engineering and Technology, pp. $1-10$. 